UAHEP-9603

DSFNA-T-9606

ESI-310

February 1996

\title{
Integrability of the Wess-Zumino-Witten model as a non-ultralocal theory
}

\author{
${ }^{*}$ S. G. Rajeev日, ${ }^{* *}$ A. Sternf and ${ }^{* * *}$ P. Vitale \\ The Erwin Schrödinger Institute for Mathematical Physics \\ Pasteur gasse 6/7, A-1090 Wien Austria \\ * Department of Physics, University of Rochester \\ Rochester, NY 14627 USA \\ **Department of Physics, University of Alabama, Tuscaloosa, AL 35487 USA \\ *** Dipartimento di Scienze Fisiche, Università di Napoli \\ Mostra d'Oltremare, Pad.19 - 80125 Napoli, ITALY
}

\begin{abstract}
We consider the 2-dimensional Wess-Zumino-Witten (WZW) model in the canonical formalism introduced in [2]. Using an $r-s$ matrix approach to non-ultralocal field theories we find the Poisson algebra of monodromy matrices and of conserved quantities with a new, non-dynamical, $r$ matrix.
\end{abstract}

\footnotetext{
${ }^{\dagger}$ rajeev@urhep.pas.rochester.edu

$\ddagger$ astern@ua1vm.ua.edu

$\S$ vitale@axpna1.na.infn.it
} 


\section{Introduction}

In the last decades a large class of 2-dimensional solvable models in field theory has been well established both at the classical and at the quantum level, via the inverse scattering method; for a comprehensive review see [1] and references therein. The distinguishing feature of such solvable models is the existence of a couple of matrices (a Lax pair), which are functions of the fields and of a spectral parameter, and which linearize the equations of motion. Such matrices satisfy a Poisson algebra which is ultralocal, which means that it doesn't contain derivatives of the delta function. It is this property which ensures the existence of a well defined Poisson bracket for monodromy matrices, the Jacobi identity for such an algebra being insured by the Yang-Baxter equation for an $r-$ matrix.

Ultralocality also implies that the $r$ matrix is non-dynamical (i.e., it doesn't depend on space-time coordinates). As a result an infinite subset of conserved quantities of the theory is in involution.

Unfortunately interesting theories like the WZW model are non-ultralocal. As was shown in [5], non-ultralocality introduces discontinuous functions in the Poisson brackets of monodromy matrices, making it difficult to have a well defined algebra of conserved quantities; also, the Jacobi identity does not follow anymore from a Yang-Baxter equation. Nonetheless many attempts have been made to solve theories of this kind and solutions have been proposed for different models [国, [5], [6], [13], [14], [15], [8], [9], [10], [11], [12].

In this paper we follow the approach contained in [4] where an extended Yang-Baxter type algebra for the monodromy matrices is established and conditions for the conserved quantities to be in involution are given.

\section{Canonical formalism for the WZW model and as- sociated linear system}

In [2] a new canonical formalism for the WZW model was given. In this section we first sketch the conventional description, then summarize the results found in [0] which will be used in the paper, and introduce the Lax pair for our theory.

The action which describes the model is

$$
S=\frac{1}{4 \chi^{2}} \int d^{2} x \operatorname{Tr}\left(\partial_{\mu} g \partial_{\nu} g^{-1}\right) \eta^{\mu \nu} \sqrt{-\operatorname{det}(\eta)}+n \Gamma
$$


where $g: R^{1,1} \longrightarrow S U(2)$ is a map from the two-dimensional Minkowski space to the simple compact Lie group $S U(2), \eta$ is the Minkowskian metric, $\Gamma$ is the WZW action

$$
\Gamma=\frac{1}{24 \pi} \int_{B} d^{3} y \epsilon^{i j k} \operatorname{Tr} g^{-1} \partial_{i} g g^{-1} \partial_{j} g g^{-1} \partial_{k} g
$$

and $B$ is a 3 -dimensional manifold whose boundary is the space-time. The coefficient of the WZW action is defined as

$$
\rho=\frac{n \chi^{2}}{4 \pi}
$$

The equations of motion are

$$
\partial^{\mu}\left(\partial_{\mu} g g^{-1}\right)-\rho \epsilon^{\mu \nu} \partial_{\mu} g g^{-1} \partial_{\nu} g g^{-1}=0
$$

The crucial property of this model is that it can be formulated entirely in terms of the current $\partial_{\mu} g g^{-1}$. The equation of motion (4) is equivalent to the following pair of first order equations,

$$
\begin{aligned}
& \frac{\partial I}{\partial t}=\frac{\partial J}{\partial x}+\rho[I, J] \\
& \frac{\partial J}{\partial t}=\frac{\partial I}{\partial x}-[I, J]
\end{aligned}
$$

The second equation is the integrability condition for the existence of $g: R^{1,1} \longrightarrow S U(2)$ satisfying,

$$
\begin{aligned}
I & =\frac{\partial g}{\partial t} g^{-1} \\
J & =\frac{\partial g}{\partial x} g^{-1}
\end{aligned}
$$

$I$ and $J$ are traceless antihermitean matrices valued in the Lie algebra of $S U(2)$

$$
I=I_{\alpha}(x) \frac{i}{2} \sigma^{\alpha}, \quad J=J_{\alpha}(x) \frac{i}{2} \sigma^{\alpha}
$$

where $\sigma^{\alpha}$ are the Pauli matrices. If we also impose the boundary condition

$$
\lim _{x \rightarrow-\infty} g(x)=1
$$

the solution for $g$ is unique. Then equation (5) guarantees that $g$ satisfy the equation of motion (11).

Though (5) can be obtained from an action principle, the Hamiltonian formalism is more suitable for our purposes; the Hamiltonian and Poisson brackets which give (5) are then

$$
H_{1}=-\frac{1}{2 \chi^{2}} \int \operatorname{tr}\left(I^{2}+J^{2}\right) d x
$$




$$
\begin{aligned}
\frac{1}{2 \chi^{2}}\left\{I_{\alpha}(x), I_{\beta}(y)\right\}_{1} & =\varepsilon_{\alpha \beta \gamma} I_{\gamma}(x) \delta(x-y)+\rho \varepsilon_{\alpha \beta \gamma} J_{\gamma} \delta(x-y), \\
\frac{1}{2 \chi^{2}}\left\{I_{\alpha}(x), J_{\beta}(y)\right\}_{1} & =\varepsilon_{\alpha \beta \gamma} J_{\gamma}(x) \delta(x-y)-\delta_{\alpha \beta} \delta^{\prime}(x-y), \\
\frac{1}{2 \chi^{2}}\left\{J_{\alpha}(x), J_{\beta}(y)\right\}_{1} & =0 ;
\end{aligned}
$$

where $\varepsilon_{\alpha \beta \gamma}$ are the structure constants of $S U(2) . I_{\alpha}, J_{\alpha}$ defined by (9) are square integrable functions on the space time; the square integrability is a condition on how quickly the currents must decay to zero at infinity. It is needed for finiteness of energy (see $(11))$.

As can be easily checked, the Poisson algebra given above is the semidirect sum of an abelian algebra and a Kac-Moody algebra associated to $S U(2)$.

The same equations of motion can be obtained from an alternative Hamiltonian formalism [2], where the new Hamiltonian and Poisson brackets depend on an extra parameter $\tau$, the limit $\tau \rightarrow 0$ being the conventional formalism. The alternative Hamiltonian and Poisson brackets are respectively:

$$
\begin{gathered}
H=-\frac{1}{2 \chi^{2}\left(1-\tau^{2}\right)^{2}} \int \operatorname{tr}\left(I^{2}+J^{2}\right) d x \\
\frac{1}{2 \chi^{2}}\left\{I_{\alpha}(x), I_{\beta}(y)\right\}=\left(1-\tau^{2}\right) \varepsilon_{\alpha \beta \gamma} I_{\gamma}(x) \delta(x-y) \\
+a\left(1-\tau^{2}\right) \varepsilon_{\alpha \beta \gamma} J_{\gamma}(x) \delta(x-y) \\
\frac{1}{2 \chi^{2}}\left\{I_{\alpha}(x), J_{\beta}(y)\right\}=\left(1-\tau^{2}\right) \varepsilon_{\alpha \beta \gamma} J_{\gamma}(x) \delta(x-y) \\
+\left(1-\tau^{2}\right)^{2} \delta_{\alpha \beta} \delta^{\prime}(x-y) \\
+\left(1-\tau^{2}\right) \epsilon \varepsilon_{\alpha \beta \gamma} I_{\gamma}(x) \delta(x-y) \\
\frac{1}{2 \chi^{2}}\left\{J_{\alpha}(x), J_{\beta}(y)\right\}=\tau^{2}\left(1-\tau^{2}\right) \varepsilon_{\alpha \beta \gamma} I_{\gamma}(x) \delta(x-y) \\
+\left(1-\tau^{2}\right) \mu \varepsilon_{\alpha \beta \gamma} J_{\gamma}(x) \delta(x-y),
\end{gathered}
$$

where $a, \mu, \epsilon$ are real parameters depending on $\tau$ as we will state below; $\tau$ can be chosen either real or imaginary as both the Hamiltonian and the Poisson brackets only depend on $\tau^{2}$. Let us call this algebra $\mathcal{C}_{2}$. It can be verified that we get the correct equations of motion, (5), if we pose

$$
\rho=\frac{a-\epsilon}{1-\tau^{2}}
$$

The Poisson algebra $\mathcal{C}_{2}$ can be rewritten in a much simpler way if we perform the change of variables:

$$
\begin{aligned}
I & =2 \chi^{2}\left(1-\tau^{2}\right)(\alpha L+\beta R) \\
J & =2 \chi^{2}\left(1-\tau^{2}\right)(\gamma L+\delta R)
\end{aligned}
$$


with $L$ and $R$ generators of two commuting Kac-Moody algebras,

$$
\begin{aligned}
\left\{L_{\alpha}(x), L_{\beta}(y)\right\}_{2} & =\varepsilon_{\alpha \beta \gamma} L_{\gamma}(x) \delta(x-y)+\frac{k}{2 \pi} \delta_{\alpha \beta} \delta^{\prime}(x-y) \\
\left\{R_{\alpha}(x), R_{\beta}(y)\right\}_{2} & =\varepsilon_{\alpha \beta \gamma} R_{\gamma}(x) \delta(x-y)-\frac{\bar{k}}{2 \pi} \delta_{\alpha \beta} \delta^{\prime}(x-y) \\
\left\{L_{\alpha}(x), R_{\beta}(y)\right\}_{2} & =0
\end{aligned}
$$

and $k, \bar{k}$ are a pair of constants. Note that the change of variables to $L$ and $R$ is singular if $\tau=0$. It is now straightforward (although quite tedious) to show that this algebra goes over to $\mathcal{C}_{2}$ under the above change of variables, if we choose

$$
\begin{array}{rlrl}
\alpha=1-\rho \tau & \beta=1+\rho \tau \\
\gamma=\tau(\rho \tau-1) & \delta=\tau(\rho \tau+1) \\
\epsilon=\mu=\rho \tau^{2} & a=\rho \\
k=\frac{\pi}{2 \chi^{2} \tau(1-\rho \tau)^{2}} & \bar{k}=\frac{\pi}{2 \chi^{2} \tau(1+\rho \tau)^{2}} .
\end{array}
$$

Thus our current algebra $\mathcal{C}_{2}$ is isomorphic to a direct sum of two commuting Kac-Moody algebras, when $\tau$ is real. We can conclude that the alternative canonical formalism for the WZW model here sketched is equivalent to the conventional one but has the advantage of exhibiting a Poisson algebra which is a sum of two Kac-Moody algebras, $\widehat{\mathcal{L S U}}(2) \oplus \widehat{\mathcal{L S U}}(2)$ if $\tau$ is real; if we choose $\tau$ to be imaginary it can be checked that $\mathcal{C}_{\in}$ is instead the Kac-Moody algebra associated to $S L(2, C), \widehat{\mathcal{L S}} \mathcal{L}(2, C)$ (this is due to the fact that $I$ and $J$ become complex combinations of the $\widehat{\mathcal{L S U}}(2) \oplus \widehat{\mathcal{L S U}}(2)$ generators, when $\tau$ is imaginary). We will assume from now on $\tau$ real, the procedure being identical for $\tau$ imaginary.

The WZW model admits an associated linear system [1]

$$
\begin{aligned}
\partial_{x} \psi(x, t, \lambda) & =A(x, t, \lambda) \psi(x, t, \lambda) \\
\partial_{t} \psi(x, t, \lambda) & =M(x, t, \lambda) \psi(x, t, \lambda),
\end{aligned}
$$

where $(A, M)$ is the so called Lax pair,

$$
\begin{aligned}
A(x, t, \lambda) & =\frac{1}{2} I\left\{\frac{1-\rho}{1-\lambda}-\frac{1+\rho}{1+\lambda}\right\}+\frac{1}{2} J\left\{\frac{1-\rho}{1-\lambda}+\frac{1+\rho}{1+\lambda}\right\} \\
M(x, t, \lambda) & =\frac{1}{2} I\left\{\frac{1-\rho}{1-\lambda}+\frac{1+\rho}{1+\lambda}\right\}+\frac{1}{2} J\left\{\frac{1-\rho}{1-\lambda}-\frac{1+\rho}{1+\lambda}\right\} .
\end{aligned}
$$

Because of their dependence on the currents, $A$ and $M$ are traceless matrices valued in the Lie algebra of $S U(2)$ if the spectral parameter $\lambda$ is real, or in the Lie algebra of $S L(2, C)$ if we choose $\lambda$ to be complex. It is convenient to rewrite the Lax pair in terms of the new basis that we have chosen for the current algebra,

$$
L(x, t)=\frac{\tau I-J}{4 \chi^{2} \tau\left(1-\tau^{2}\right)(1-\rho \tau)}, \quad R(x, t)=\frac{\tau I+J}{4 \chi^{2} \tau\left(1-\tau^{2}\right)(1+\rho \tau)},
$$


so that

$$
\begin{aligned}
A(x, t, \lambda) & =a(\lambda) L(x, t)+b(\lambda) R(x, t) \\
M(x, t, \lambda) & =c(\lambda) L(x, t)+d(\lambda) R(x, t),
\end{aligned}
$$

with

$$
\begin{aligned}
& a(\lambda)=\chi^{2}\left(1-\tau^{2}\right)(1-\rho \tau)\left[\frac{1-\rho}{1-\lambda}(1-\tau)-\frac{1+\rho}{1+\lambda}(1+\tau)\right] \\
& b(\lambda)=\chi^{2}\left(1-\tau^{2}\right)(1+\rho \tau)\left[\frac{1-\rho}{1-\lambda}(1+\tau)-\frac{1+\rho}{1+\lambda}(1-\tau)\right] \\
& c(\lambda)=\chi^{2}\left(1-\tau^{2}\right)(1-\rho \tau)\left[\frac{1-\rho}{1-\lambda}(1-\tau)+\frac{1+\rho}{1+\lambda}(1+\tau)\right] \\
& d(\lambda)=\chi^{2}\left(1-\tau^{2}\right)(1+\rho \tau)\left[\frac{1-\rho}{1-\lambda}(1+\tau)+\frac{1+\rho}{1+\lambda}(1-\tau)\right] .
\end{aligned}
$$

The compatibility condition of equations (28) and (29) for any value of $\lambda$, which reads

$$
\partial_{t} A-\partial_{x} M+[A, M]=0
$$

implies the equations of motion, (5) and (6). Relation (36) is also known as zero curvature condition for the connection $(M, A)$.

We define the monodromy matrix $T(x, y, \lambda)$ (the $\mathrm{t}$ dependence being understood from now on), as a particular solution of (28), (29):

$$
\begin{aligned}
& \partial_{x} T(x, y, \lambda)=A(x, t, \lambda) T(x, y, \lambda) \\
& \partial_{t} T(x, y, \lambda)=M(x, t, \lambda) T(x, y, \lambda)-T(x, y, \lambda) M(y, t, \lambda),
\end{aligned}
$$

with

$$
\begin{gathered}
T(x, x, \lambda)=1 \quad T(y, x, \lambda)=T^{-1}(x, y, \lambda) \\
T(x, y, \lambda) T(y, z, \lambda)=T(x, z, \lambda) .
\end{gathered}
$$

We have then

$$
T(x, y ; \lambda)=\mathrm{P} \exp \int_{y}^{x} A\left(x^{\prime}, \lambda\right) d x^{\prime}
$$

where $\mathrm{P}$ denotes path ordering. The infinite volume limit of $T(x, y, \lambda)$

$$
T(\infty,-\infty ; \lambda) \equiv T(\lambda)=P \exp \int_{-\infty}^{\infty} A(x, \lambda) d x
$$

is a conserved quantity for any value of $\lambda$. In fact from (38) we have:

$$
\partial_{t} T(\infty,-\infty ; \lambda)=\partial_{t}[M(\infty, \lambda) T(\lambda)-T(\lambda) M(-\infty, \lambda)]
$$


which is zero, because

$$
\lim _{x \rightarrow \pm \infty} I(x)=\lim _{x \rightarrow \pm \infty} J(x)=0
$$

and $M$ a linear function of the currents $I, J$. Note that for periodic boundary conditions only the trace of $T(\lambda)$ is conserved.

From the definition of $T(\lambda)$ we note that $T(\lambda)$ (as a function of $\lambda$ ) is an element of the loop group of $S L(2, \mathbb{C})$; in fact $A(\lambda= \pm \infty)=0$ thus implying

$$
T(\lambda=-\infty)=T(\lambda=\infty)=1 .
$$

Also it satisfies

$$
T^{\dagger}(\lambda) T(\bar{\lambda})=1 .
$$

The last equation is easily proven observing that $A^{\dagger}(x, \lambda)=-A(x, \bar{\lambda})$ If we choose the spectral parameter to be real (42) implies that $T(\lambda)$ be an element of the loop group of $S U(2)$.

Because $T(\lambda)$ is an ordered exponential of $A(x, \lambda)$, we only need the Poisson algebra of the $A^{\prime} s$ to determine the Poisson bracket of the monodromy matrices. From (33), (34), and the Poisson brackets of the Kac-Moody generators we get

$$
\begin{aligned}
\left\{A^{a}(x, \lambda), A^{b}(y, \mu)\right\} & =\varepsilon_{a b c}\left(g(\lambda, \mu) A^{c}(x, \lambda)+g(\mu, \lambda) A^{c}(y, \mu)\right) \delta(x-y) \\
& -f(\lambda, \mu) \delta_{a b} \delta^{\prime}(x-y)
\end{aligned}
$$

where

$$
\begin{aligned}
g(\lambda, \mu) & =a(\mu) b(\mu) \frac{a(\lambda)-b(\lambda)}{a(\lambda) b(\mu)-a(\mu) b(\lambda)} \\
g(\mu, \lambda) & =-a(\lambda) b(\lambda) \frac{a(\mu)-b(\mu)}{a(\lambda) b(\mu)-a(\mu) b(\lambda)} \\
f(\lambda, \mu) & =b(\lambda) b(\mu) \frac{\bar{k}}{2 \pi}-a(\lambda) a(\mu) \frac{k}{2 \pi} .
\end{aligned}
$$

We pose

$$
\hat{g}(\lambda, \mu)=g(\lambda, \mu) \mathrm{C}, \quad \hat{g}(\mu, \lambda)=g(\mu, \lambda) \mathrm{C}, \quad \hat{f}(\lambda, \mu)=f(\lambda, \mu) \mathrm{C}
$$

where $\mathrm{C}=\frac{1}{4} \sigma_{a} \otimes \sigma^{a}$. Defining then $r(\lambda, \mu)$ and $s(\lambda, \mu)$ as the skew-symmetric and the symmetric part of $\hat{g}(\lambda, \mu)$, respectively,

$$
\begin{aligned}
& r(\lambda, \mu)=\frac{1}{2}(\hat{g}(\lambda, \mu)-\hat{g}(\mu, \lambda)) \\
& s(\mu, \lambda)=\frac{1}{2}(\hat{g}(\lambda, \mu)+\hat{g}(\mu, \lambda)),
\end{aligned}
$$

and observing that

$$
\hat{f}(\lambda, \mu)=2 s(\lambda, \mu)
$$


we can rewrite $(43)$ as

$$
\begin{gathered}
\left\{A_{1}(x, \lambda), A_{2}(y, \mu)\right\}=\left(\left[r(\lambda, \mu), A_{1}(x, \lambda)+A_{2}(x, \mu)\right]\right. \\
\left.-\left[s(\lambda, \mu), A_{1}(x, \lambda)-A_{2}(x, \mu)\right]\right) \delta(x-y)-2 s(\lambda, \mu) \delta^{\prime}(x-y),
\end{gathered}
$$

where $A=A^{a} \frac{i}{2} \sigma_{a}, A_{1}=A \otimes 1, A_{2}=1 \otimes A$. The matrices $r, s$, as explicit functions of $\lambda$ and $\mu$, are

$$
\begin{aligned}
r & =2 \chi^{2} \frac{\left(1-\tau^{2}\right)}{\lambda-\mu}\left\{\frac{\lambda^{2}\left(1-\rho^{2} \tau^{2}\right)-2 \lambda \rho\left(1-\tau^{2}\right)+\rho^{2}-\tau^{2}}{\lambda^{2}-1}\right. \\
& \left.+\frac{\mu^{2}\left(1-\rho^{2} \tau^{2}\right)-2 \mu \rho\left(1-\tau^{2}\right)+\rho^{2}-\tau^{2}}{\mu^{2}-1}\right\} \mathrm{C} \\
s & =2 \chi^{2} \frac{\left(1-\tau^{2}\right)}{\lambda-\mu}\left\{\frac{\lambda^{2}\left(1-\rho^{2} \tau^{2}\right)-2 \lambda \rho\left(1-\tau^{2}\right)+\rho^{2}-\tau^{2}}{\lambda^{2}-1}\right. \\
& \left.-\frac{\mu^{2}\left(1-\rho^{2} \tau^{2}\right)-2 \mu \rho\left(1-\tau^{2}\right)+\rho^{2}-\tau^{2}}{\mu^{2}-1}\right\} \mathrm{C} .
\end{aligned}
$$

In the limit $\rho=0, \tau=0$, which is the chiral model in the usual formalism the $r$ and $s$ matrices given above reduce to the ones found in [6] by general arguments on Poisson brackets related to Kac-Moody algebras. Models which are described by a Poisson algebra containing derivatives of the delta function are called non-ultralocal [1]. In general they also exhibit a space-time dependence for $r$ and $s$ matrices [4], [5], the general form of the Poisson algebra thus being:

$$
\begin{gathered}
\left\{A_{1}(x, \lambda), A_{2}(y, \mu)\right\}=\left(\partial_{x} r(x, \lambda, \mu)+\left[r(x, \lambda, \mu), A_{1}(x, \lambda)+A_{2}(x, \mu)\right]\right. \\
\left.-\left[s(x, \lambda, \mu), A_{1}(x, \lambda)-A_{2}(x, \mu)\right]\right) \delta(x-y)-(s(x, \lambda, \mu)+s(y, \lambda, \mu)) \delta^{\prime}(x-y) .
\end{gathered}
$$

In principle there could be higher derivatives of the delta function. Note however that in our case, though the system is non-ultralocal, the $r$ and $s$ matrices are non-dynamical. The Poisson bracket (50) has a remarkable property: it is well defined for any value of $\tau$, even for the singular value $\tau=0$, where the Kac-Moody generators, $\mathrm{L}, \mathrm{R}$, are not independent functions of $I, J$.

\section{Poisson algebra of monodromy matrices and equal- point limits}

In this section we summarize the procedure to obtain Poisson brackets for monodromy matrices starting from Poisson brackets of the currents $A(x, \lambda)$; we also find the equal-

"note that for $\rho=0, \tau \neq 0$ we have a family of principal chiral models depending on an extra parameter $\tau[3]$. 
point limit and verify that our $r$ and $s$ matrices satisfy a sort of Yang-Baxter equation (see [5]) which is the Jacobi identity for non-ultralocal theories. For the ultralocal case a detailed description is given in [1].

The Poisson algebra for monodromy matrices is obtained from the algebra (50) in the following way:

$$
\begin{gathered}
\left\{T_{a b}(x, y, \lambda), T_{c d}\left(x^{\prime}, y^{\prime}, \mu\right)\right\}= \\
\int_{y}^{x} \int_{y^{\prime}}^{x^{\prime}} d z d z^{\prime} \frac{\delta T_{a b}(x, y, \lambda)}{\delta A_{i j}(z, \lambda)}\left\{A_{i j}(z, \lambda), A_{k l}\left(z^{\prime}, \mu\right)\right\} \frac{\delta T_{c d}\left(x^{\prime}, y^{\prime}, \mu\right)}{\delta A_{k l}\left(z^{\prime}, \mu\right)}= \\
\int_{y}^{x} \int_{y^{\prime}}^{x^{\prime}} d z d z^{\prime} T_{a i}(x, z, \lambda) T_{j b}(z, y, \lambda)\left\{A_{i j}(z, \lambda), A_{k l}\left(z^{\prime}, \mu\right)\right\} T_{c k}\left(x^{\prime}, z^{\prime}, \mu\right) T_{l d}\left(z^{\prime}, y^{\prime}, \mu\right),
\end{gathered}
$$

where we used

$$
\delta T(x, y, \lambda)=\int_{y}^{x} T(x, z, \lambda) \delta A(z, \lambda) T(z, y, \lambda) d z .
$$

Using tensorial formalism and the notation $T_{1}=T \otimes 1, T_{2}=1 \otimes T$ we can rewrite (54) as

$$
\begin{gathered}
\left\{T_{1}(x, y, \lambda), T_{2}\left(x^{\prime}, y^{\prime}, \mu\right)\right\}= \\
\int_{y}^{x} \int_{y^{\prime}}^{x^{\prime}} d z d z^{\prime} T_{1}(x, z, \lambda) T_{2}\left(x^{\prime}, z^{\prime}, \mu\right)\left\{A_{1}(z, \lambda), A_{2}\left(z^{\prime}, \mu\right)\right\} T_{1}(z, y, \lambda) T_{2}\left(z^{\prime}, y^{\prime}, \mu\right) .
\end{gathered}
$$

Substituting (50) and performing the integral we get

$$
\begin{gathered}
\left\{T_{1}(x, y, \lambda), T_{2}\left(x^{\prime}, y^{\prime}, \mu\right)\right\}= \\
T_{1}\left(x, x_{0}, \lambda\right) T_{2}\left(x^{\prime}, x_{0}, \mu\right)\left(r(\lambda, \mu)+\epsilon\left(x-x^{\prime}\right) s(\lambda, \mu)\right) T_{1}\left(x_{0}, y, \lambda\right) T_{2}\left(x_{0}, y^{\prime}, \mu\right)- \\
T_{1}\left(x, y_{0}, \lambda\right) T_{2}\left(x^{\prime}, y_{0}, \mu\right)\left(r(\lambda, \mu)+\epsilon\left(y-y^{\prime}\right) s(\lambda, \mu)\right) T_{1}\left(y_{0}, y, \lambda\right) T_{2}\left(y_{0}, y^{\prime}, \mu\right),
\end{gathered}
$$

where $\epsilon(x)=\operatorname{sign}(x), x_{0}=\min \left(x, x^{\prime}\right), y_{0}=\max \left(y, y^{\prime}\right)$. We note that the Poisson brackets (57) have been found under the explicit assumption that $x, x^{\prime}, y, y^{\prime}$, be all different; also this algebra shows up a discontinuity of amplitude $2 s$, in the equal-point limits $x=x^{\prime}, y=y^{\prime}$.

The Jacobi identity for the Poisson brackets (57)

$$
\left\{T_{1}(x, y, \lambda),\left\{T_{2}\left(x^{\prime}, y^{\prime}, \mu\right), T_{3}\left(x^{\prime \prime}, y^{\prime \prime}, \nu\right)\right\}\right\}+\text { cycl perm }=0
$$

results in an equation for the $r$ and $s$ matrices:

$$
\begin{gathered}
{\left[(r-s)_{12}(\lambda, \mu),(r+s)_{13}(\lambda, \nu)\right]+} \\
{\left[(r+s)_{12}(\lambda, \mu),(r+s)_{23}(\mu, \nu)\right]+\left[(r+s)_{13}(\lambda, \nu),(r+s)_{23}(\mu, \nu)\right]=0 .}
\end{gathered}
$$


If $r$ and $s$ depend on space-time variables, equation (59) will also contain terms involving the Poisson bracket of $r$ and $s$ with $A$. As can be seen (59) reduces to the usual YangBaxter equation for the $r$ matrix when $s$ is zero. This is the ultralocal case.

It can be checked that our $r$ and $s$ matrices given in (51) and (52) satisfy condition (59), which can also be rewritten as an equation for the matrix $g$. Noting that

$$
\hat{g}(\lambda, \mu)=r(\lambda, \mu)+s(\lambda, \mu), \quad-\hat{g}(\mu, \lambda)=r(\lambda, \mu)-s(\lambda, \mu)
$$

equation (59) becomes then

$$
\begin{gathered}
-\left[\hat{g}_{12}(\mu, \lambda), \hat{g}_{13}(\lambda, \nu)\right]+ \\
+\left[\hat{g}_{12}(\lambda, \mu), \hat{g}_{23}(\mu, \nu)\right]+\left[\hat{g}_{13}(\lambda, \nu), \hat{g}_{23}(\mu, \nu)\right]=0 .
\end{gathered}
$$

It can be verified, as a consistency check, that the same condition is obtained from the Jacobi identity of the current algebra (50).

\subsection{Equal-point limits of the monodromy algebra}

The monodromy matrices $T(x, y, \lambda)$ evaluated at $x=\infty, y=-\infty$ are conserved quantities for any value of $\lambda$ as shown with (41). We want to show that there are functions of them which are in involution with respect to the Poisson brackets (57). To do this, we have first to define the Poisson brackets (57) for equal points $x=x^{\prime}, y=y^{\prime}$. As already noted they are discontinuous at those points so that we cannot simply put $x=x^{\prime}, y=y^{\prime}$. There are many regularization procedures to define such limit. We follow here a symmetric limit procedure illustrated in [5] which we briefly summarize in the following. We pose

$$
\Delta^{(1)}\left(x_{1}, x_{2} ; y_{1}, y_{2} ; \lambda_{1}, \lambda_{2}\right)=\left\{T_{1}\left(x_{1}, y_{1}, \lambda_{1}\right), T_{2}\left(x_{2}, y_{2}, \lambda_{2}\right)\right\}
$$

then the equal point limit $x_{1}=x_{2}=x$ is defined as:

$$
\Delta^{(1)}\left(x, x ; y_{1}, y_{2} ; \lambda_{1}, \lambda_{2}\right)=\lim _{\epsilon \rightarrow 0} \frac{1}{2 !} \sum_{\sigma} \Delta^{(1)}\left(x+\epsilon \sigma(1), x+\epsilon \sigma(2) ; y_{1}, y_{2} ; \lambda_{1}, \lambda_{2}\right),
$$

where the sum is over the permutations of $\{1,2\}$. Analogously we can define the limit $y_{1}=y_{2}=y$, so that the Poisson bracket for two monodromy matrices at equal spacetime points is

$$
\left\{T_{1}(x, y, \lambda), T_{2}(x, y, \mu)\right\}=\Delta^{(1)}(x, x ; y, y ; \lambda, \mu) .
$$

The introduction of the tensor $\Delta$ is particularly useful if generalized to n-nested Poisson brackets. In particular we are interested to rewrite the Jacobi identity using such a notation; we define

$$
\Delta^{(2)}\left(x_{i}, x_{j}, x_{k} ; y_{i}, y_{j}, y_{k} ; \lambda_{i}, \lambda_{j}, \lambda_{k}\right)=\left\{T_{i}\left(x_{i}, y_{i}, \lambda_{i}\right),\left\{T_{j}\left(x_{j}, y_{j}, \lambda_{j}\right), T_{k}\left(x_{k}, y_{k}, \lambda_{k}\right)\right\}\right\},
$$


so that the symmetric limit $x_{1}=x_{2}=x_{3}$ is

$$
\begin{gathered}
\Delta^{(2)}\left(x, x, x ; y_{i}, y_{j}, y_{k} ; \lambda_{i}, \lambda_{j}, \lambda_{k}\right)= \\
\lim _{\epsilon \rightarrow 0} \frac{1}{3 !} \sum_{\sigma} \Delta^{(2)}\left(x+\epsilon \sigma(1), x+\epsilon \sigma(2), x+\epsilon \sigma(3) ; y_{1}, y_{2}, y_{3} ; \lambda_{i}, \lambda_{j}, \lambda_{k}\right) .
\end{gathered}
$$

Using (64) to define the equal point limits for the monodromy matrices algebra we get

$$
\left\{T_{1}(x, y, \lambda), T_{2}(x, y, \mu)\right\}=\left[r(\lambda, \mu), T_{1}(x, y, \lambda) T_{2}(x, y, \mu)\right]
$$

Note that this Poisson bracket has the same form of the Poisson bracket obtained for ultralocal models but it satisfies Jacobi identity only through the symmetric limit procedure (65). Let us see this in more detail.

Jacobi identity for (66) is defined through the symmetric limit procedure (65) as:

$$
\Delta^{(2)}(x, y ; \lambda, \mu, \nu)+\Delta^{(2)}(x, y ; \nu, \lambda, \mu)+\Delta^{(2)}(x, y ; \mu, \nu, \lambda)=0 .
$$

This identity is implied from (58) (or equivalently (59)). Each term

$$
\Delta^{(2)}\left(x, y ; \lambda_{i}, \lambda_{j}, \lambda_{k}\right)=
$$

$\lim _{\epsilon_{1}, \epsilon_{2} \rightarrow 0} \sum_{\sigma, \tilde{\sigma}} \Delta^{(2)}\left(x+\epsilon_{1} \sigma(1), x+\epsilon_{1} \sigma(2), x+\epsilon_{1} \sigma(3) ; y+\epsilon_{2} \tilde{\sigma}(1), y+\epsilon_{2} \tilde{\sigma}(2), y+\epsilon_{2} \tilde{\sigma}(3) ; \lambda_{i}, \lambda_{j}, \lambda_{k}\right)$

is the sum of 36 terms, 6 for each choice of the x's configuration, so that we have 108 terms. They combine three by three in Jacobi identities of the kind (58):

$$
\begin{gathered}
\left\{T_{1}\left(x+\epsilon_{1} \sigma(1), y+\epsilon_{2} \tilde{\sigma}(1), \lambda_{i}\right),\left\{T_{2}\left(x+\epsilon_{1} \sigma(2), y+\epsilon_{2} \tilde{\sigma}(2), \lambda_{j}\right), T_{3}\left(x+\epsilon_{1} \sigma(3), y+\epsilon_{2} \tilde{\sigma}(3), \lambda_{k}\right)\right\}\right\} \\
+ \text { cycl perm }=0
\end{gathered}
$$

because (58) is satisfied for each choice of $x \neq x^{\prime} \neq x^{\prime \prime}, y \neq y^{\prime} \neq y^{\prime \prime}$. Put

$$
\begin{gathered}
J_{\sigma(1) \sigma(2) \sigma(3)}= \\
\sum_{\tilde{\sigma}} \Delta^{(2)}\left(x+\epsilon_{1} \sigma(1), x+\epsilon_{1} \sigma(2), x+\epsilon_{1} \sigma(3) ; y+\epsilon_{2} \tilde{\sigma}(1), y+\epsilon_{2} \tilde{\sigma}(2), y+\epsilon_{2} \tilde{\sigma}(3) ; \lambda_{i}, \lambda_{j}, \lambda_{k}\right)
\end{gathered}
$$

(which we have just shown to be zero term by term); Jacobi identity for (66) reads then

$$
\sum_{\sigma} J_{\sigma(1) \sigma(2) \sigma(3)}=0
$$

If we check Jacobi identity directly on (66) we find a Yang-Baxter equation for the $r$-matrix given in (51), which is not satisfied. For this reason we say that (66) satisfies Jacobi identity only in a weak sense. 
In the infinite volume limit, equation (66) reads

$$
\left\{T_{1}(\lambda), T_{2}(\mu)\right\}=\left[r(\lambda, \mu), T_{1}(\lambda) T_{2}(\mu)\right],
$$

and the conserved quantities, $\operatorname{Tr} T(\lambda)$, are in involution, being $\operatorname{Tr}(A \otimes B)=\operatorname{Tr} A \cdot \operatorname{Tr} B$, so that

$$
\{\operatorname{Tr} T(\lambda), \operatorname{Tr} T(\mu)\}=\operatorname{Tr}\left\{T_{1}(\lambda), T_{2}(\mu)\right\},
$$

which is zero because of $(\sqrt{70})$, being the trace of a commutator. Note that the Poisson algebra

$$
\{\operatorname{Tr} T(\lambda), \operatorname{Tr} T(\mu)\}=0
$$

satisfies Jacobi identity stronlgly (which is trivially true for zero Poisson brackets).

\section{Conclusions}

We have found a three-parameter family of non-ultralocal integrable models (the parameters being $\tau, \rho$, and the coupling constant $\chi$ ). The Poisson algebra of monodromy matrices can be rewritten in terms of $r$ and $s$ matrices which are independent on spacetime variables and satisfy an extended Yang-Baxter equation. We also exhibit the conserved quantities of the theory which are in involution with respect to such a Poisson structure.

\section{Acknowledgements}

We wish to thank Giuseppe Marmo and Peter Michor for hospitality at ESI where this work was done. A.S. was supported in part by the Department of Energy, USA, under contract number DE-FG05-84ER40141. 


\section{REFERENCES}

[1] L. D. Faddeev, L.A. Takhtajan Hamiltonian methods in the theory of solitons Springer Verlag Ed. (1987) and refs. therein.

[2] S. G. Rajeev, G. Sparano and P. Vitale Int J. of Mod. Phys. A31 (1994) 5469

[3] S. G. Rajeev Phys. Lett. B217 (1989) 123

[4] J. M. Maillet Phys. Lett. B162 (1985) 137

[5] J. M. Maillet Nucl. Phys. B269 (1986) 54

[6] J. M. Maillet Phys. Lett. B167 (1986) 402

[7] L. Friedel and J. M. Maillet Phys. Lett B262 (1991) 278

[8] B. G. Konopelchenko Rev. in Math. Phys 2 (1990) 399

[9] L. Hlavaty J. Math. Phys 36 (1995) 4883

[10] O. Babelon and L. Bonora Phys. Lett B253 (1991) 365

[11] L. Hlavaty and A. Kundu Quantum integrability of non local models through Baxterization of quantized braided algebras Preprint. hep-th 9406215 .

[12] M. Semenov Tian-Shansky and A. Sevostyanov Classical and quantum nonultralocal systems on the lattice Preprint. hep-th 9509029.

[13] M. C. B. AbdallaPhys. Lett. B152 (1985) 215

[14] E. Abdalla, M. C. B. Abdalla, J. C. Brunelli and A. Zadra Comm. Math. Phys. 166 (1994) 379

[15] E. Abdalla and M. Forger Mod. Phys. Lett. A7 (1992) 2437 